\title{
El proyecto de vida de los jóvenes: sus valores, expectativas, motivaciones y elementos de sentido ${ }^{1}$
}

\author{
Paula Leiva Sandoval*
}

\author{
RESUMEN
}

El presente artículo muestra los resultados de una investigación correspondiente a un estudio de caso, que se centró en la comprensión de los Proyectos de Vida de un grupo de jóvenes, a través del reconocimiento en sus discursos de los elementos fundamentales con los que construyen sus anhelos de futuro.

Con la intención de acceder a la realidad juvenil se aplicó la semántica estructural, para desde una mirada cualitativa conocer más profundamente a ese sujeto joven, que frente a los profundos cambios sociales, culturales y económicos globales, y a la presión de las expectativas y las dificultades del diario vivir que debe enfrentar, se repliega a su espacio más íntimo para construir su Proyecto de Vida.

Palabras clave: Juventud / Proyecto de Vida / Discurso juvenil / búsqueda personal/semántica estructural de Greimas.

\section{O projecto de vida dos jovens: seus valores, expectativas, motivações e elementos de sentido}

\author{
RESUMO
}

O presente artigo mostra os resultados de uma investigação correspondente a um estudo de caso, que se centrou no entendimento dos Projectos de Vida de um grupo de jovens, através do reconhecimento em seus discursos dos elementos fundamentais com os que constroem seus anseios de futuro.

Com a intenção de aceder à realidade juvenil aplicou-se a semántica estrutural, para desde uma mirada cualitativa conhecer mais profundamente a esse sujeito jovem, que em frente às profundas mudanças sociais, culturais e económicos globais, e à pressão das expectativas e as dificuldades do diário viver que deve enfrentar, se repliega a seu espaço mais íntimo para construir seu Projecto de Vida

1 Este artículo corresponde a una síntesis de la investigación realizada, entre agosto del 2008 y mayo del 2009, por Carlos Oyarce, Nelson Segovia y Paula Leiva, en el marco de la Tesis de grado del Magíster en Trabajo Social "Competencias para la Acción Socioeducativa" de la Universidad Tecnológica Metropolitana de Chile (Leiva, et al. 2009).

* Chilena, Trabajadora Social. Magíster en Trabajo Social. Docente de la Escuela de Trabajo Social de la Universidad Católica Silva Henríquez. Correo electrónico: pleiva@ucsh.cl 
Palavras chave: Juventude / Projecto de Vida / Discurso juvenil / busca pessoal /semántica estrutural de Greimas

\section{The project of life of the young: his values, expectations, motivations and elements of sense}

ABSTRACT

The present article shows the results of a corresponding investigation to a study of case, that centered in the understanding of the Projects of Life of a group of young, through the recognition in his speeches of the fundamental elements with which build his anhelos of future.

With the intention to access to the juvenile reality applied the semantic structural, stop from a look cualitativa know more deeply to this young subject, than front to the deep social changes, cultural and economic global, and to the pressure of the expectations and the difficulties of the daily live that it has to enfrentar, repliega to his more intimate space to build his Project of Life.

Key Words: Youth / Project of Life / juvenile Speech / semantic personal / research structural of Greimas. 


\section{Antecedentes}

Al abordar la temática juvenil, es posible observar que existen hasta hoy diversos autores y numerosos estudios e investigaciones que presentan el fenómeno de la juventud desde múltiples perspectivas. Se hace necesario, por lo tanto, en este ejercicio de aproximación, referirse a las "juventudes", aludiendo a la diversidad de aspectos que caracterizan hoy en día a los jóvenes.

En tal sentido y con la intención de acceder a la realidad juvenil desde una mirada distinta, se incorporaron a la investigación elementos que permitieron conocer más profundamente a ese sujeto joven, que frente a los profundos cambios sociales, culturales y económicos globales, y a la presión de las expectativas y las dificultades del diario vivir, que debe enfrentar, se repliega a su espacio más íntimo para ir construyendo su Proyecto de Vida.

En torno a este último concepto, Proyecto de Vida, se articuló la investigación desarrollada. Para ello fue necesario dar cuenta de los diversos aportes teóricos que abordan la temática juvenil, pero que recogieran en ese análisis, aspectos relacionados con la visión de futuro que tienen los jóvenes y las influencias que ejerce el medio social en la construcción de esa imagen futura.

Cobra sentido, en relación a lo último, lo planteado por Klaudio Duarte (2007), cuando señala que es necesario ejercitarse en mirar caleidoscópicamente el mundo, la vida y los sueños que presentan los jóvenes, para así reconocerlos en su complejidad. Sugiriendo, realizar una aproximación a la realidad juvenil, que considera una lectura profunda de los contextos sociales, culturales, políticos y económicos, en los que se insertan los jóvenes hoy.

Chile presenta en las últimas décadas un acelerado proceso de cambio cultural que se aprecia, por ejemplo, en los medios de comunicación, nuevas formas de consumo, nuevas tecnologías, lo que según Baeza (2003) ha hecho que los valores y símbolos tradicionales con que las familias, personas y grupos orientan su vida, se vean difusos. Este cambio cultural está influyendo en la forma cómo las personas viven sus vidas y construyen sus capacidades individuales y sus proyectos vitales.

Los efectos de este cambio tienen un impacto especial en los jóvenes, quienes viven con intensidad los procesos de construcción de sus proyectos vitales, para lo cual requieren de 
un referente social, que les permita apoyarse en su proceso de crecimiento y desarrollo.

La juventud, desde esta perspectiva, es considerada como una etapa de preparación para el futuro, donde la calidad de la educación recibida y la voluntad personal se constituyen en elementos clave para el logro de una "buena vida", tal y como lo señalan los jóvenes y lo confirman los resultados de la V Encuesta Nacional de Juventud (2007). En estos términos, se podría afirmar que el futuro se forja con buena educación -como movilizador social- y con perseverancia y esfuerzo personal.

Los resultados de la V Encuesta Nacional de Juventud (INJUV, 2007) permiten hablar hoy en día de un período existencial asociado a la etapa juvenil, de trayectorias no lineales dado que cada joven vive esta condición de distinta forma.

Esta trayectoria vital se va construyendo con aquello que vamos concretando, en relación a nuestros anhelos y deseos personales. Luego de un ejercicio reflexivo, y como lo señala Atria (1993), proyectamos nuestra mirada hacia el futuro y nos vemos desempeñándonos profesionalmente en distintos ámbitos y asumiendo diversos roles en estructuras familiares y sociales. Lo anterior se constituye en el material con que se construye el mundo de los proyectos de vida.

Es a través de la acción imaginativa que podemos vernos como sujetos de ciertos roles; nos percibimos reflexivamente como el actor que creemos que vamos a ser en ese futuro. Esta construcción de futuro personal se hace sobre la base de las expectativas.

El mundo de los proyectos de vida se construye, según Atria (1993), como una serie de apuestas a lo probable; la afirmación de un estado de cosas futuro que se nos presenta como relativamente seguro y próximo. La concreción de los proyectos de vida se realiza frecuentemente por medio de un ajuste, a veces muy costoso en términos personales, de las expectativas que son corregidas por el realismo. Nos vemos a nosotros mismos como el sujeto que creemos que vamos a ser en un futuro próximo. "Cuando yo sea tal o cual cosa..." es el tipo de expresión que encontramos en el eje semántico del lenguaje que utilizamos para referirnos a nosotros mismos en relación al propio proyecto de vida.

La construcción del Proyecto de Vida personal, según lo señala D’Angelo (1998), se vincula también a la formación de la identidad 
de la persona, que se concibe como un proceso complejo de construcción de la personalidad desde los primeros años de vida, pero este proceso transcurre en un contexto sociocultural específico, a través de la mediación de los adultos y la influencia de normas y patrones sociales definidos. Por tanto, la formación de la identidad social es el marco en el que se configuran las identidades individuales.

El Proyecto de Vida articula la identidad personal-social en las perspectivas de su dinámica temporal y posibilidades de desarrollo futuro. Se comprende, entonces, como un sistema principal de la persona en su dimensionalidad esencial de la vida. Es un modelo ideal sobre lo que el individuo espera o quiere ser y hacer, que toma forma concreta en la disposición real y sus posibilidades internas y externas de lograrlo, definiendo su relación hacia el mundo y hacia sí mismo, su razón de ser como individuo en un contexto y tipo de sociedad determinada (D’Angelo, 1998).

\section{Aproximación a los proyectos de vida de los jóvenes}

Para alcanzar el objetivo principal de este estudio, centrado en la comprensión de los Proyectos de vida de un grupo de jóvenes, buscando relevar desde su propia experiencia los elementos fundamentales, que éstos integran en la construcción de sus proyectos vitales, como asimismo para conocer los valores, las expectativas, las motivaciones y los elementos de sentido que subyacen en sus discursos, se diseñó una investigación con enfoque cualitativo, utilizando la semántica estructural como método de análisis de los relatos. Se otorgó un énfasis central al discurso de los actores y su visión de proceso, recogiendo los significados que los sujetos de la investigación asignan a su Proyecto de Vida. La investigación contempló una muestra de 17 jóvenes entre 17 y 24 años, pertenecientes a distintas carreras (Trabajo Social, Pedagogía en Educación Física, Pedagogía en Educación Básica, Pedagogía en Matemáticas, Pedagogía en Educación Diferencial, Pedagogía en Castellano y Pedagogía en Filosofía) de la Universidad Católica Silva Henríquez de Santiago de Chile, con quienes se realizaron tres grupos de discusión y tres entrevistas en profundidad.

El material textual recogido a través de tales técnicas, fue analizado con el método de la semántica estructural desarrollado 
por Greimas. Entre los conceptos que Greimas propone y aplica en su modelo de análisis semántico, se encuentran aspectos centrales como los referidos al signo lingüístico, a su naturaleza y a sus relaciones con otros signos semánticos.

En todo proceso de representación simbólica interviene un sujeto sometido a reglas y leyes culturales, sociales y simbólicas, propias de su grupo de origen, de su clase, de su comunidad y de su entorno natural de vida.

Por lo anterior, tenemos a un sujeto (S) que se ubica frente a un objeto $(\mathrm{O})$, el que -a su vez- está condicionado por otros sujetos (S`) que alteran sustancialmente sus características, en un medio ambiente dado.

Consecuente con lo anterior, se puede señalar que todo texto, como actualización de un modelo codificado, produce significado a través de la peculiaridad de la estructura de los actantes de su relato. De esta manera, la estructura actancial bajo la forma de "relato de búsqueda" con la cual se trabajó, es la siguiente:

Cuadro $\mathrm{N}^{\circ}$ 1: Fuente: Baeza et al. (2009)

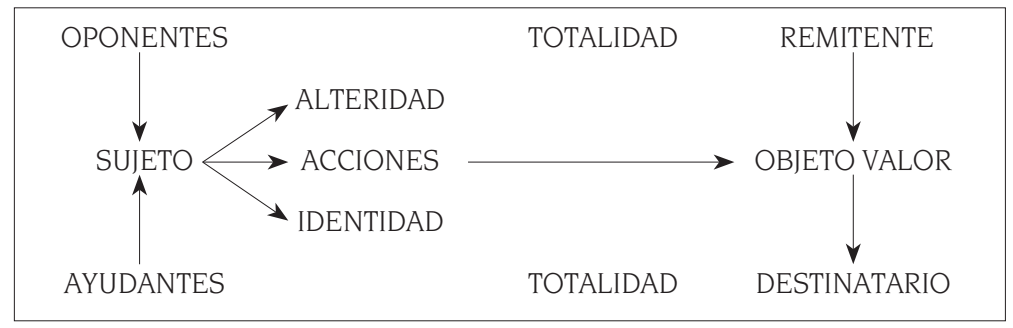

En el esquema del relato de búsqueda el Sujeto (S), a partir de una carencia, de una necesidad y/o de una expectativa, emprende la búsqueda de un Objeto $(O)$ que satisfará esa carencia, necesidad y/o expectativa. Este objeto surge de un proceso de comunicación que va desde un Remitente (Rem.), que es el productor simbólico del objeto, o proveedor de la competencia simbólica, para que efectúe el proceso de búsqueda, que envía al Objeto hacia un actante que es el Destinatario (Dest.)

En la trama del relato de esa búsqueda, el Sujeto se enfrenta a ciertas pruebas calificantes o descalificantes, los que podríamos llamar los límites que tiene para el logro de sus expectativas. En ese contexto, el Sujeto se enfrenta a Adversarios u Oponentes (Op) y cuenta con ciertos ayudantes (Ay) para conseguir sus fines. En el proceso de "relato de búsqueda", el sujeto desarrolla 
una "identidad", entendida como el conjunto de características específicas que le otorgan la individualidad al sujeto, diferenciándolo de los otros.

Al mismo tiempo, el sujeto (S) se enfrenta a un otro diferente, a un "alter" que en términos semánticos, es definido como "alteridad" del objeto, es decir, el hecho de ser otro, de reconocer la diferencia a través de rasgos característicos diferentes. La relación que se establece entre el Sujeto y su "alter" (entre Identidad y Alteridad) toma cuerpo en un contexto determinado, el que, en términos semióticos, ha sido definido como "totalidad".

\section{Relato de Búsqueda de los jóvenes}

Los relatos de los Proyectos de Vida de los jóvenes entrevistados fueron abordados, como se señaló anteriormente, con el método de análisis de la semántica estructural. Concluidos los análisis y descripciones de los relatos en torno a los proyectos de vida de los sujetos, según sus campos lexemáticos, a continuación se presenta la forma en que se constituyen los mundos de los proyectos de vida, a través de sus relatos.

La estructura actancial es la siguiente:

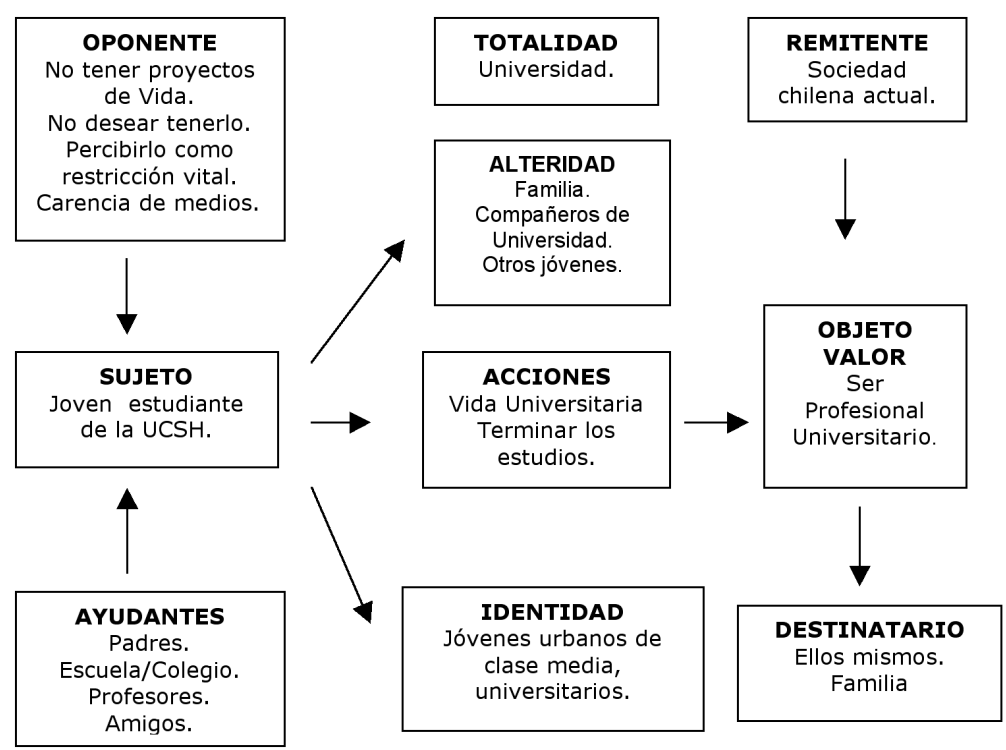

Al analizar los relatos de los estudiantes entrevistados, podemos identificar al sujeto semántico: el Joven estudiante de la UCSH. Es precisamente este joven el que realiza acciones en pos de 
un objeto valor, que estaría dado por el deseo manifestado por los entrevistados de ser un Profesional Universitario, una vez concluida la carrera.

Este objeto valor es el producto de una demanda que es transversal a todos los grupos sociales, incluso cuando las condiciones socioeconómicas son muy precarias, ya que la Educación Superior es vista, por la sociedad y también por los jóvenes, como un medio concreto de movilidad social y se percibe como una verificación del éxito y el crecimiento personal.

Las acciones que este sujeto emprende para conseguir su objeto valor, es precisamente cumplir su proceso de formación en el tiempo determinado para su carrera, desarrollando una vida universitaria que implica cumplir con las tareas académicas que se desprenden de la formación, asistir regularmente a clases, apropiarse de los contenidos específicos de la disciplina estudiada, participar en las tareas grupales, incorporándose a la vida estudiantil, desarrollando la disciplina personal, acciones concretas que le permitirán conseguir su objeto que es no solo terminar sus estudios, sino que además conseguir el título universitario.

Para lograr su cometido, el joven cuenta con personas concretas que colaboran en este proceso. Según el relato de los jóvenes, estos ayudantes, estarían dados en un primer lugar por sus padres, quienes los motivaron para estudiar, y en la mayoría de los casos, son quienes financian los estudios superiores y mantención de los jóvenes, brindando el apoyo económico necesario.

En el discurso de los jóvenes, también aparece la familia como un todo, es decir, es vista como el principal soporte afectivo y el eje estructurante de la personalidad.

En otro lugar como ayudantes del logro del objeto, se ubica la Escuela o Colegio de origen, ya que la formación recibida en ese espacio, tanto en la enseñanza básica como la enseñanza media, es determinante, según relatan los jóvenes, al momento de acceder a la educación superior. Según los antecedentes revisados, efectivamente el prestigio del establecimiento educacional de donde provengan los jóvenes, además de otros factores que favorecen el que la experiencia educativa sea significativa, como por ejemplo el vínculo que se establece entre el educador y alumno, son altamente influyentes, ya que contribuyen, entre otros factores, a elevar la autoestima y a generar expectativas superiores de desarrollo personal. 
Un tercer ayudante, y relacionado con el anterior, son los Profesores. La figura del profesor se señala como modelo a seguir, especialmente en los jóvenes que estudian pedagogía. La experiencia significativa o el vínculo generado con un profesor en la etapa de enseñanza media o básica, se muestra como determinante de la motivación para estudiar en la universidad y/o para estudiar alguna carrera relacionada con la pedagogía.

Un cuarto ayudante, además del anterior, señalado por los jóvenes, son los amigos, quienes se constituyen en el grupo donde los jóvenes encuentran distracción, afecto y apoyo. Este cuarto ayudante, como grupo de semejantes, se constituyen en un referente afectivo importante para los jóvenes, especialmente en los momentos difíciles. Es un espacio o ámbito de la vida personal, donde se comparten generacionalmente, explicaciones para la vida, se transmite información, se busca reconocimiento de sí mismo en otros, que son un igual, se experimentan vivencias y situaciones de vida comunes y se accede a códigos y símbolos propios de la etapa vital en la que se encuentran.

Todas las acciones descritas habilitan al joven para el proceso de desligamiento de la familia como grupo inicial de referencia, constituyéndose el grupo de amigos, en un espacio de autoafirmación que lo prepara para la vida adulta.

De modo que es posible reconocer ayudantes en el relato de los jóvenes, en cuanto a la consecución del objeto valor que es ser profesional, también es posible reconocer a los oponentes, es decir a aquello que impide o dificulta la obtención del objeto valor. En un primer lugar, reconocemos como oponente, el hecho de que los jóvenes manifiesten "no tener proyectos de vida". Por lo tanto, algunos de ellos no reconocen el estar estudiando una carrera profesional, como un elemento o una acción que favorezca la construcción de sus proyectos de vida personal, sino más bien lo perciben como un hecho puntual, sin ser parte de un plan predeterminado por ellos mismos. Algunos de los entrevistados plantean el no desear tener un proyecto de vida, porque desde su mirada la planificación le resta espontaneidad a la vida, percibiendo la construcción de un proyecto de vida como una restricción vital.

En tal sentido, podríamos afirmar que estos jóvenes que no desean tener un proyecto de vida, podrían no estar interesados en planificar su proyecto vital, porque este ejercicio anticipatorio, los enfrenta a un futuro que los adultos y la sociedad en su 
conjunto, no los convence ni los invita. Ya que en el futuro se ubican las posibilidades tanto de inserción laboral como social y esta idea de inserción no es atrayente porque perciben que sus proyectos de vida podrían verse obstaculizados por condiciones socio-estructurales que afectan a algunos sectores de la población como impedimentos de pleno acceso a mejores oportunidades.

Frente a estas opiniones, podríamos afirmar que a los y las jóvenes el tema del futuro les preocupa, ya que éste se presenta, en algunas ocasiones, como incierto e inestable. Esta percepción poco esperanzadora del futuro, está en cierta forma influenciada por las señales que les llegan desde el mundo adulto.

De igual forma la carencia de medios, también se percibe como un oponente a la obtención del objeto valor, medios que entre otros se refieren a los económicos que son la capacidad de pagar los estudios, la mantención del estudiante, los gastos permanentes asociados al trabajo académico y todo aquello que aporta para continuar con los estudios.

En los relatos de los jóvenes, también es posible reconocer un Remitente, es decir, desde dónde el sujeto toma la idea de lo importante y valioso que es el objeto valor a conseguir. En el caso de los jóvenes entrevistados, se observa como remitente la sociedad chilena actual, debido a que el hecho de Ser profesional se constituye en un anhelo generalizado de muchos jóvenes, por cuanto, como se ha dicho anteriormente, es percibido como un medio concreto de movilidad social y como un aspecto importante para ser y sentirse feliz.

También se reconoce, al interior de los relatos de los jóvenes, un Destinatario, es decir hacia dónde se dirige el objeto valor. En este caso se aprecia a los mismos jóvenes como destinatarios de su objeto. Por cuanto el Ser profesional traería, según lo afirman en sus relatos, consecuencias positivas para la propia vida y la de quienes los rodean, que en el caso de los jóvenes entrevistados, estarían constituidos por sus familias de origen y aquel núcleo familiar que quisieran constituir en el futuro.

Este destinatario es un sujeto en construcción que se encuentra planteándose metas y trayectos que se han de seguir para lograr los objetivos personales. En otras palabras, el joven se plantea su proyecto de vida no como un mañana incierto, sino más bien, como un presente que vivencia intensamente y le permite caminar 
hacia esa meta-futuro desde el presente. El logro de esa meta, según el relato de los jóvenes, no solo beneficiará al joven, sino que también a la familia que acompaña cercanamente el proceso de formación de su hijo.

En cuanto a la identidad de los sujetos, es decir, aquellas características que los identifican como grupo social, se perciben como jóvenes de clase media, estudiantes universitarios, de sectores urbanos de la población. Asimismo, relevan en la mayoría de los casos, ser la primera generación al interior de sus familias de origen que tienen la posibilidad de acceder a la educación superior. En tal sentido, y según lo que relatan los jóvenes, ellos se perciben como parte de un grupo privilegiado al acceder a la educación superior, ya que ésta posibilidad es vista como una oportunidad concreta de movilidad social, que va en beneficio no solo del propio joven, sino también de sus familias.

La alteridad que se percibe en su relatos está dada principalmente por aquellos que son referidos recurrentemente en sus relatos, estos son la familia de origen, como la instancia en donde encuentran el apoyo afectivo y también económico; los compañeros de universidad con los que comparten intereses comunes y con aquellos que se habilitan en el trabajo colaborativo. Y los otros jóvenes, de los cuales se diferencian, por haber tenido la posibilidad de acceder a la educación superior.

Todo lo anterior se presenta en una totalidad, que se constituye en el contexto; en el caso de los jóvenes entrevistados, éste estaría dado por la universidad, como el espacio común donde se construyen las vivencias personales de estos jóvenes. La Universidad, no solo percibida como espacio que da cuenta de una infraestructura puesta al servicio de los procesos formativos que se viven al interior, si no como la comunidad de personas que conviven en ella, constituida por los académicos, personal de gestión y los estudiantes.

En cuanto a los estudiantes que fueron entrevistados, comparten con el resto de los estudiantes de la Universidad Católica Silva Henríquez, la etapa vital en la que se encuentran, es decir, jóvenes que han optado por integrarse a un proceso de formación que les permitirá desarrollarse personal y profesionalmente, para constituirse, en un futuro cercano, en profesionales, objeto de cada uno de los estudiantes de esta Universidad. 


\section{Conclusiones}

La comprensión de los Proyectos de Vida de los jóvenes, entrega algunos elementos comunes que es posible relevar del discurso juvenil, en el sentido de que comparten un conjunto de valores, motivaciones, metas, expectativas que declaran para su futuro personal.

Este proyecto vital se construye, en el habla juvenil, en el día a día para algunos, mientras que para otros responde a una motivación más profunda del "siempre quise ser" o se explicita a través de un camino o trayecto que se va haciendo a medida que se va creciendo, en el sentido de la madurez personal, frente a las vivencias que van conformando el bagaje individual, con el cual enfrenta tanto la búsqueda de identidad personal como social.

La búsqueda también presenta otras connotaciones, aunque de manera infrecuente, que en el léxico juvenil, tan claro como contradictorio, nos señala que lo que parece tan claro y adecuado, como tener un proyecto de vida, es decir planificar el futuro, no es más que restar espontaneidad a la vida, por lo que adquiere una calidad definida como restricción vital.

Concluimos entonces que las y los jóvenes, conscientes de la etapa vital en que se encuentran, abordan el horizonte existencial que se despliega ante ellos, con una construcción que se anida en su espacio más íntimo, replegada frente a los profundos cambios sociales y culturales que los presionan. Sin embargo, la oportunidad de brindarles un espacio para poder expresar ese conjunto de expectativas y sueños, o temores y rebeldías, también nos señala que hay un espacio vacío entre el presente, ser joven, y el futuro, ser adulto, que bien pudiera estar sembrado de temores y angustias que no se explicitan, pero que claramente se perciben desde un mundo adulto que no los atrae.

Para algunos autores, el proyecto de vida no se entiende solo en función del logro de metas, sino relevando la importancia del trayecto que se ha de seguir para lograrlas. En otras palabras, visualizar el proyecto de vida no como un mañana incierto, sino como presente vivido con intensidad y profundidad para construir desde el hoy.

\section{En relación a los valores}

Frente a los valores, entendidos como una creencia, acerca de que un modo de existencia es personal y socialmente preferible 
a otros modos de conducta, las y los jóvenes, mayoritariamente, expresan preocupación por el desarrollo y superación personal, como asimismo responsabilidad por la transmisión de valores fundamentales en el contacto derivado del trabajo con las personas

Lo anterior da cuenta de que efectivamente las y los jóvenes confían en su propio esfuerzo y autodeterminación más que en otros aspectos exteriores tales como los familiares, los pares o la comunidad, lo que entendemos vinculado a los procesos de individuación que actualmente experimenta la sociedad chilena. Uno de cuyos aspectos dice relación con la desconfianza hacia las instituciones que promueven la participación social. Sin embargo, paradójicamente, crece el interés de los jóvenes por las actividades solidarias.

En el relato de los jóvenes, es posible reconocer aquellos aspectos a los cuales ellos le otorgan un valor fundamental en sus vidas. Al reflexionar respecto a lo que quieren para su vida en el futuro, es decir al realizar ese ejercicio de planificación, enfatizan principalmente un deseo de crecimiento y desarrollo personal permanente, es decir, a lo largo de toda su existencia.

Subyace aquí una paradoja respecto a lo planteado en relación al proceso de individuación que se está experimentando en nuestra sociedad, ya que los niveles de desarrollo que quieren alcanzar los jóvenes durante su proceso vital no están pensados de manera exclusiva para ellos mismos; los jóvenes ven en esta posibilidad de desarrollo un beneficio que tocará también a quienes los rodean, especialmente a sus familias, pero no excluyen de los beneficios de este desarrollo al resto de la sociedad.

Sienten que el tener oportunidades en la vida los responsabiliza de tener que "devolver la mano", vislumbrándose en ellos un profundo sentido de responsabilidad social. Están convencidos de que una vez que participen del mundo adulto, asumiendo las responsabilidades que de esa etapa vital se derivan, les corresponderá realizar su aporte a la sociedad, desde el lugar que cada uno ocupe en ella.

Esto último se encuentra influido en un primer nivel por la vivencia y los afectos experimentados al interior de sus familias; son ellos los que, según sus relatos, les han inculcado el valor de preocuparse por aquellos socialmente desfavorecidos. Sin embargo, este valor no solo se instaló desde la experiencia 
familiar, sino también fue reforzado en la formación recibida durante la Educación Superior.

Es posible reconocer en la institución de la cual provienen los jóvenes entrevistados, una formación fuertemente orientada al servicio y la trasformación de las realidades sociales injustas. Por lo tanto, los valores recibidos al interior de las familias desde la más temprana infancia y durante la experiencia escolar posterior, se articulan de manera especial, en la instancia de la formación profesional, instalándose en los jóvenes como un sólido valor que constituye un elemento fundamental en la construcción del proyecto de vida personal.

Al decir de los autores consultados, hoy día se vivencia un proceso de grandes transformaciones culturales desde un modelo centrado en la razón social a uno centrado en la autorrealización autónoma, del cual dan cuenta los discursos juveniles como se expresó precedentemente. Sin embargo, es importante relevar del habla juvenil el frecuente uso de términos valóricos asociados a una disposición personal de servicio, donde la preocupación y responsabilidad por los otros es manifiesta.

Es importante señalar que estos procesos de cambio cultural también acarrean tensiones en cuanto a la construcción de las identidades juveniles asociadas a proyectos de vida, que si bien aparecen viables en el habla juvenil, también se enfrentan a los problemas personales de las y los jóvenes, como son la carencia de medios socio - económicos que les impedirían su total realización.

Es posible concluir, en el ámbito de los valores, que las y los jóvenes expresan con mayor fuerza aquellos valores que se vinculan con la profesionalidad, el compromiso social y los asociados a la satisfacción de las necesidades materiales. Y que construyen sus proyectos de vida con una óptica en el marco de la individuación, pero con un profundo contenido valórico de respeto al otro y responsabilidad, asociada a lo que será su desempeño laboral como profesionales en el futuro.

\section{En relación a las expectativas}

En cuanto a las expectativas, vistas éstas como una suposición centrada en el futuro, podemos señalar que los jóvenes entrevistados poseen una visión optimista del futuro. Al imaginarse proyectivamente en el tiempo, en el corto plazo se 
visualizan terminando los estudios, y en el mediano plazo se imaginan ejerciendo la profesión para la cual se están formando en la actualidad y conformando una familia.

Reconocen en el hecho de haber tenido la oportunidad de cursar estudios superiores, que esto los ubica en una posición de ventaja por sobre muchos jóvenes que no tuvieron (y no tienen actualmente), la posibilidad de acceder a la educación superior.

Perciben este tiempo de formación como una etapa preparatoria para el ingreso a la vida adulta, centrando sus esfuerzos en el desarrollo de sus potencialidades y en la búsqueda de espacios de crecimiento, considerando que todas estas acciones les permitirán insertarse en la sociedad de una mejor forma en un futuro próximo.

En este aspecto, se observó una nítida diferenciación asociada al género, ya que son las jóvenes las que mencionan prioritariamente, dentro de sus proyecciones personales, la constitución de una familia.

\section{En relación a las motivaciones}

Las motivaciones son lo que promueve, mantiene y dirige las acciones de un sujeto o sujetos, orientado a una meta u objetivos que se desean alcanzar. Tienen una función dinamizadora del comportamiento humano, otorgándole un sentido de proyección futura. En nuestro análisis con la metodología de la semántica estructural, se afirma que en el relato de búsqueda, el Sujeto (S), a partir de una carencia, de una necesidad y/o de una expectativa, emprende la búsqueda de un objeto valor (OV) que satisfará esa carencia, necesidad y/o expectativa.

Esta motivación tras un logro, que en nuestro caso es la obtención de un título universitario, es denominado en el lenguaje corriente como amor propio, espíritu de superación o de lucha y se caracteriza, entre otros aspectos, por su propositividad, es decir, tiene una clara orientación a obtener lo deseado, y es persistente en el tiempo.

En el caso de las y los jóvenes consultados en este estudio, la principal motivación que presentan, durante esta etapa de formación en la educación superior en la que se encuentran, es principalmente concluir con éxito los estudios, para así obtener el tan ansiado título universitario. Sin embargo, desde 
los relatos juveniles es posible recoger otras motivaciones asociadas a esta última, que dice relación con la estabilidad laboral y económica que desearían tener una vez egresados de la educación superior.

Es posible concluir entonces que las motivaciones que se aprecian en estos jóvenes sí promueven, mantienen y dirigen las acciones, ya que el discurso explicita que existe total claridad en la meta a perseguir y además nos indica que estas motivaciones son tanto intrínsecas, propias de los sujetos, como extrínsecas, es decir hay agentes externos que han cooperado en su realización, que son identificados en el discurso juvenil, como los padres, los profesores y los amigos cercanos.

\section{En relación a los elementos de sentido}

Son aquellos aspectos que le permiten al joven delinear un rumbo para su vida, con una visión optimista de ésta. Es decir, es el resultado de un descubrimiento personal, donde se reconoce la libertad para construir la propia historia, es decir, la capacidad que tiene la persona de ir forjando su propia vida y su propio futuro, a través de sus actos y decisiones.

Desde los relatos de los jóvenes es posible identificar claramente algunos aspectos que podrían constituirse en elementos de sentido para su vida. El más importante es el "ser feliz", lo que se vincula con la dimensión ética del ser humano. El ser humano es ético porque se construye a sí mismo a través de sus actos. Y con sus actos transforma su realidad para que ésta sea como él quiere.

El otro elemento de sentido se centra en los ideales, y se alude nuevamente con esto, a la dimensión ética de la persona, ya que estos ideales junto al proyecto de vida se constituyen en elementos que le permiten al ser humano trascender la realidad actual y lo orientan en la perspectiva de su futuro.

Los jóvenes al reflexionar respecto a su Proyecto de Vida están señalando ideales, es decir, anhelos de realización o de lo que quieren llegar a ser en el futuro. Cuando los jóvenes plantean que desean "devolver la mano", están aludiendo a un anhelo que no concluye en sí mismos, es decir, trasciende la propia persona del joven, refiriéndose al deseo de aportar, desde lo que cada joven es y será en el futuro, a una sociedad mejor. 
Con todo lo anterior, es posible señalar que la construcción del Proyecto de vida no es un ejercicio privativo de la etapa juvenil, ya que los adultos a lo largo de su existencia, también deben revisar y replantear sus proyectos vitales. Sin embargo, es en la etapa juvenil, donde se sientan las bases de ese proyecto, es el momento donde se toman las decisiones más importantes y que determinan en gran medida el rumbo de la existencia de la persona.

En tal sentido, es posible señalar que los estudiantes consultados en este estudio se encuentran en una etapa vital determinante, ya que están definiendo lo que quieren llegar a ser en el futuro desde la perspectiva profesional.

Al adentrarnos en los Proyectos vitales de los jóvenes, es posible constatar que el momento o etapa en la que se encuentran está llena de desafíos, que en ocasiones les provocan sentimientos de inseguridad y temor. En su mayoría están conscientes de que el futuro no se puede improvisar y que cada una de las decisiones que tomen en este momento, tendrá efectos y consecuencias para su porvenir, y ese solo hecho los asusta.

Es posible señalar que en este punto radica la negación, que algunos jóvenes plantearon, de no desear tener un proyecto de vida, porque ese ejercicio de planeación de la propia vida, implica para ellos tomar decisiones que aún no han reflexionado profundamente. Por lo tanto, no quisieran correr el riesgo de proponerse algo que no puedan lograr, evitando así la sensación de fracaso que pudieran sentir en el futuro.

La juventud es una etapa de profundos cambios, de dejar atrás lo que se fue y el momento en que surgen muchas interrogantes sobre lo que se llegará a ser. En tal sentido, es posible observar al joven en una búsqueda permanente de aquellos elementos que le permitirán convertirse y darle forma a su "propio adulto", apoyándose, como se ha señalado en esta investigación, en las relaciones que establece con sus padres, profesores, sus pares y otros adultos significativos, que a través de mecanismos de identificación, darán forma a su propio inventario personal de valores, creencias, ideales y sentidos que articulan y articularán el desarrollo de su vida.

Es posible afirmar entonces que la construcción del Proyecto de Vida en los jóvenes se da en una dualidad de Acción - Decisión. Los jóvenes se ven enfrentados a esta dualidad, a tener que 
definir hacia dónde orientarán su vida. Esta acción implica para ellos, correr riesgos, cometer errores, experimentar en ocasiones el fracaso; pero también implica aprender, crear y desarrollarse en la acción. De esta forma, se hace emerger lo mejor de la persona; el enfrentar permanentemente retos y desafíos, obligan a la persona y al joven, en este caso, a desplegar todos sus dones y capacidades personales. La construcción del Proyecto de Vida consiste en ser capaz de poner en juego lo que cada uno es, aquí y ahora, para lograr lo que se quiere para el futuro.

Este ejercicio implica riesgos, en el sentido de tener que vencer sus temores, dejar a un lado la comodidad o la estabilidad actual, para alcanzar una situación deseada en perspectiva de su futuro y así lograr las metas propuestas. Esta acción es dinámica, ya que una vez que la meta es alcanzada, el joven deberá examinar nuevos retos y desafíos a enfrentar, iniciando un nuevo ciclo, que le permitirá replantear su proyecto vital.

En relación a lo último, es posible señalar que los jóvenes con los que se desarrolló esta investigación, han dado ya un paso importante, han vencido en parte sus temores, han dejado de lado su estabilidad actual y se han propuesto cambiar; están trabajando hoy, aprovechando la oportunidad de acceso a la educación superior, para desarrollar sus talentos y ser mejores personas en el futuro, en definitiva se han desafiado personalmente a ser constructores de su propio destino. Han realizado un ejercicio de anticipación imaginaria que les permitirá vislumbrar y avanzar hacia su Proyecto de Vida futuro.

\section{Referencias Bibliográficas}

Atria, R. (1993). La educación superior desde el mundo de la vida. Revista de Estudios Sociales, 78 (4), pp. 159-177.

Baeza, Jorge (2003). Culturas Juveniles: acercamiento bibliográfico. Recuperado el 16 de Junio del 2008, de http:// bibliotecavirtual.clacso.org.ar/ar/libros/chile/ ceju/culturas.pdf

CHILE. Instituto nacional de la Juventud. (2007). Quinta Encuesta Nacional de Juventud. Santiago, Chile, INJUV.

D’Angelo, O. (1998). Proyecto de Vida como categoría básica de interpretación de la Identidad Individual y Social. Recuperado el 16 de Junio de 2008, de www.clacso.org 
Duarte Q., K. (2007). Tensiones en el análisis de lo juvenil. Revista Observatorio de Juventud, 4 (15).

Leiva, P.; Oyarce, C. y Segovia N. (2009). Proyecto de Vida en Jóvenes: Un estudio de caso. Tesis para optar al título de Magister en Trabajo Social, Escuela de Trabajo Social. Universidad Tecnológica Metropolitana UTEM, Santiago, Chile. 